\title{
Advances in design and modeling of porous materials
}

\author{
André Ayral ${ }^{a}$, Sylvie Calas-Etienne, Benoit Coasne, André Deratani, Alexis \\ Evstratov, Anne Galarneau, Daniel Grande, Matthieu Hureau, Hervé Jobic, \\ Catherine Morlay, Julien Parmentier, Bénédicte Prelot, Sylvie Rossignol, Angélique \\ Simon-Masseron, and Frédéric Thibault-Starzyk \\ Institut Européen des Membranes, CNRS-ENSCM-UM, CC047, Université de Montpellier, \\ Campus Triolet, 2 place Eugène Bataillon, 34095 Montpellier Cedex 5, France
}

This special issue of the European Physical Journal Special Topics is dedicated to selected papers from the symposium "High surface area porous and granular materials" organized in the frame of the conference "Matériaux 2014", held on November 24-28, 2014 in Montpellier, France.

Porous materials and granular materials gather a wide variety of heterogeneous, isotropic or anisotropic media made of inorganic, organic or hybrid solid skeletons, with open or closed porosity, and pore sizes ranging from the centimeter scale to the sub-nanometer scale. Their technological and industrial applications cover numerous areas from building and civil engineering to microelectronics, including also metallurgy, chemistry, health, waste water and gas effluent treatment. Many emerging processes related to environmental protection and sustainable development also rely on this class of materials. Their functional properties are related to specific transfer mechanisms (matter, heat, radiation, electrical charge), to pore surface chemistry (exchange, adsorption, heterogeneous catalysis) and to retention inside confined volumes (storage, separation, exchange, controlled release). The development of innovative synthesis, shaping, characterization and modeling approaches enables the design of advanced materials with enhanced functional performance. The papers collected in this special issue offer a good overview of the state-of-the-art and science of these complex media.

We would like to thank all the speakers and participants for their contribution to the success of the symposium. We also express our gratitude to the organization committee of "Matériaux 2014". We finally thank the reviewers and the staff of the European Physical Journal Special Topics who made the publication of this special issue possible.

\footnotetext{
${ }^{a}$ e-mail: andre.ayral@umontpellier.fr
} 